\title{
Outil d'aide à la décision dans le cadre de la maîtrise de l'aléa fontis sur la LGV Nord
}

\section{J.-L. DEHERRIPONT}

SNCF

Direction de l'ingénierie

6 , avenue

François-Mitterrand

93574 La Plaine Saint-Denis jean-luc.deherripont@sncf.fr
Entre Paris et Lille, sur les départements de l'Oise et de la Somme, le tracé de la LGV Nord traverse un environnement exposé à un aléa fontis lié pour l'essentiel à la forte concentration de vestiges militaires de la première guerre mondiale. Suite à des épisodes pluvieux d'importance centennale (1993/4 et 2001), de fortes densités de fontis ont été enregistrées dans les communes avoisinant le tracé (jusqu'à 3 fontis/ha). Depuis 1993, la gestion de l'aléa fontis sur la LGV Nord est devenue une opération importante en termes d'investigations, d'études, d'entretien, d'investissements et de surveillance des installations, générant un volume très conséquent de données. Il est apparu fondamental d'exploiter au maximum la masse des informations disponibles tout en offrant une forte réactivité en cas de découverte de fontis. Dans cet objectif, il a été décidé de s'appuyer sur les fonctionnalités de support informatique de type SIG (Système d'Information Géographique). Des développements spécifiques ont été réalisés afin d'offrir certaines fonctionnalités pour stocker, synthétiser, et mettre à jour l'information mais aussi, à terme, pour disposer d'un outil d'aide à la décision permettant d'optimiser la surveillance et la gestion de la LGV Nord soumise à l'aléa cavité.

Mots-clés : Système d'Information Géographique, gestion de risque, aléa fontis, outil d'aide à la décision.

\section{Decision-making aid tool in controlling the risk of subsidence along the Northern high speed line}

Between Paris and Lille, in the departments of the Oise and the Somme, the route of the Northern LGV line crosses a zone with a high concentration of underground vestiges, resulting predominantly from the trenches and shelters constructed during the 1st World War, few or poorly filled in. Following several severe rainfull events (1993/4 and 2001), large number of sinkholes has been observed in the aera near the route (up to 3 cases/ha). Since 1993, the management of the sinkhole hazard along the Northern LGV has become an important operation in terms of site investigations, studies, maintenance, investments and monitoring of the installations, generating an important volume of data. It appeared essential to exploit the most possible quantity of information available while being able quickly to react in case of sinkhole occurring. In this respect, it was decided to use computer tools such as GIS (Geographical Information System) to constitute a cartographic support associated with geo-refered databases. Specific developments were carried out in order to offer certain functionalities allowing to store, synthesize and update data but also, in the long term, to have a tool for decision-making processes to optimize the monitoring and the management of the Northern LGV line subjected to sinkhole hazards. 


\section{Introduction}

Entre Paris et Lille, sur les départements de l'Oise et de la Somme, le tracé de LGV Nord traverse un environnement présentant une forte concentration de vestiges souterrains, peu ou mal remblayés, résultant pour l'essentiel des tranchées et abris de la première guerre mondiale. Suite à des épisodes pluvieux d'importance centennale (1993/4 et 2001), de fortes densités de fontis ont été enregistrées dans les communes avoisinant le tracé (jusqu'à 3 fontis/ha, Figs. 1 et 2). Depuis 1993, la gestion des risques d'effondrement qui affectent la LGV Nord sur la quasi-totalité de son tracé (PK 52 à 182) est devenue une opération importante en termes d'investigations, d'études, de surveillance des installations, d'entretien et d'investissements.

Dans ce contexte, il est important que soient assemblées et synthétisées au mieux les informations relatives à ce sujet afin de bénéficier d'un retour d'expérience et d'un outil efficace pour la définition des actions à venir. L'objectif est de créer un support fonctionnel qui soit non seulement un système d'archivage dynamique permettant de stocker, de synthétiser, de traiter et de mettre à jour l'information mais aussi un outil « intelligent » d'aide à la décision pour la gestion de l'aléa cavité sur la LGV Nord.

Les systèmes d'informations géographicques ont connu ces dernières années un très vaste développement notamment dans la gestion du foncier, des réseaux et d'infrastructures. Ces outils, servis par des méthodes sérieuses d'analyse des contraintes environnementales, ont ouvert des possibilités considérables : mise en évidence des enjeux environnementaux, études multicritères, aide à la conception de projet, communication et concertation, gestion et maintenance de l'infrastructure en service. Pour toutes ces applications, le SIG a été perçu par les maîtres d'ouvrages comme une « plate-forme géomatique d'aide à la décision » (Méreau, 2002). Les SIG contribuent à améliorer la productivité des services, notamment pour tout ce qui concerne la recherche d'informations et la consultation de plans. Les avantages attendus sont aussi organisationnels, en contribuant à améliorer la coopération entre les services et en facilitant et accélérant les échanges d'informations (réactivité). Pour de telles applications, la création d'un SIG constitue un investissement important et un temps de développement relativement long avant déploiement opérationnel. Il est par conséquent important de bien mesurer au préalable les résultats attendus.

En raison de la problématique spécifique de l'aléa fontis sur la LGV Nord, il a été rapidement reconnu nécessaire tant pour RFF que pour la SNCF de disposer d'un outil permettant de valoriser les actions entreprises pour maîtriser cet aléa. La maîtrise d'ouvrage du projet a été confiée au groupement S2IF (GIE RFF-SNCF) en charge des développements de certaines applications informatiques sur le plan national. La direction de l'ingénierie SNCF a assuré le pilotage technique de l'opération et gère actuellement l'administration technique du produit (mise à jour, validation des données entrantes, interfaces utilisateurs, etc.).

\section{2}

\section{Les principaux résultats attendus du SIG}

Les données entrantes pour l'appréciation de l'aléa fontis sur le tracé de la LGV constituent autant de couches d'informations qu'il est nécessaire de juxtaposer avec un maximum de résolution afin de permettre:

- l'accès à des données contrôlées et validées avec une grande précision de localisation (les anomalies dans le sous-sol sont de petite dimension et demandent donc une résolution fine des reconnaissances depuis la surface);

- une plus crande qualité de diagnostic sur le terrain (connaissance en amont de la visite de l'historique du secteur en terme de fontis, de reconnaissances, de présence potentielle de tranchées militaires, etc.) ;

- une plus grande réactivité en cas d'apparition de fontis (rétablir au plus tôt un régime normal des circulations);

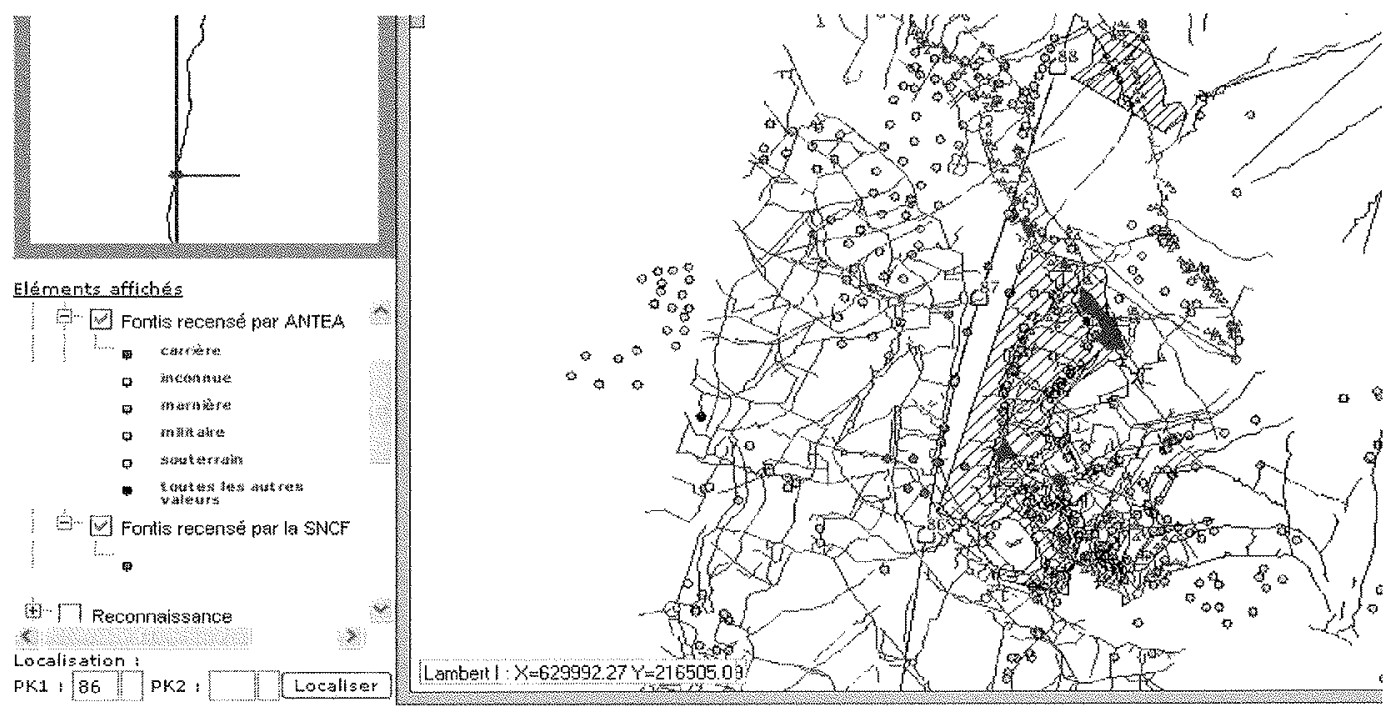




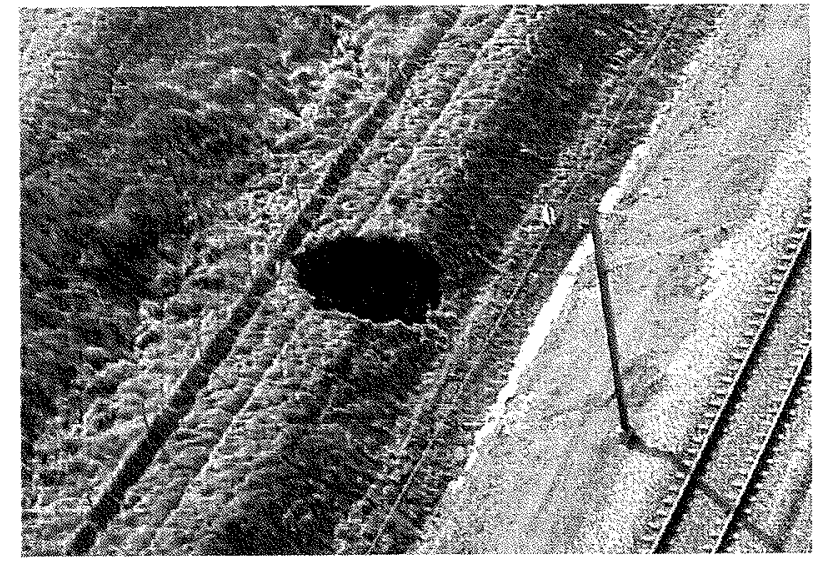

IIG. 2 Fontis dans le talus ferroviaire.

Sinkhole in the railway embankment.

- une approche multicritères des priorités d'interventions (investigations, travaux, etc.);

- une optimisation des tournées de surveillance (exploitation des observations, ajustement des seuils de déclenchement, éléments de reporting et d'amortissement des tournées, etc.).

\section{3}

\section{Inventaire des données entrantes}

Une masse considérable d'informations a été recueillie dans le cadre des actions menées pour la caractérisation et le traitement de l'aléa cavité sur la LGV Nord. L'ensemble de ces éléments contribue à la connaissance et la gestion du patrimoine sous l'angle de la maîtrise de l'aléa fontis. Au fil du temps, ces données ont été livrées sous différents supports (papier, tableur, etc.) et à différentes échelles ou sous des formes de représentation graphique différentes. Cette hétérogénéité de formats est donc peu compatible avec une exploitation et des corrélations géographiques rapides des données archivées. La création de l'outil a donc nécessité au préalable un important travail de mise en forme (uniformisation, géoréférencement, numérisation, etc.) et de vérification des données afin d'être intégrées dans l'application informatique.
Les principales couches d'informations sont les suivantes :

- fonds de plan IGN aux échelles 1/1 000, 1/5 000 et $1 / 25000$;

- inventaire des fontis depuis 1993 : bases de données incluant les fontis recensés par la SNCF dans l'environnement proche de la ligne et l'inventaire réalisé par le bureau d'études ANTEA d'après une enquête menée sur 43 communes réparties le long du tracé (Fig. 1) ;

- carte des tranchées militaires : cartographie à l'échelle 1/25 000 avec report des tranchées militaires établi à partir de récolement d'archives militaires anglaises ;

- photo-interprétation de clichés infrarouge réalisés à partir d'un vol mené en juillet 2001 au paroxysme des intempéries ; plan et photographies à l'échelle $1 / 5000$;

- cartes des anomalies résiduelles (Fig. 5) à partir des reconnaissances géophysiques en microgravimétrie (échelle 1/500), implantation des sondages réalisés suite à ces reconnaissances et base de données associée (à titre indicatif : 65000 points de mesures microgravimétriques ; 5300 sondages de 15 à $20 \mathrm{~m}$ de profondeur) ; - chantiers d'injection : plans de localisation des forages d'injection avec représentation statistique des volumes injectés.

\section{4}

\section{Les principales fonctionnalités du SIG dédié à la maîtrise de l'aléa fontis}

La base informatique s'appuie sur un outil standard (ESRI) à partir duquel des développements spécifiques ont été apportés avec l'assistance d'une société extérieure spécialisée, SWORD.

Concernant l'architecture du SIG, il est proposé un affichage à l'écran relativement classique de support SIG (Fig. 3) comprenant :

- un cadre réservé à la visualisation des supports cartographiques sélectionnés ;

- un outil de visualisation et de sélection de l'échelle de la carte ;

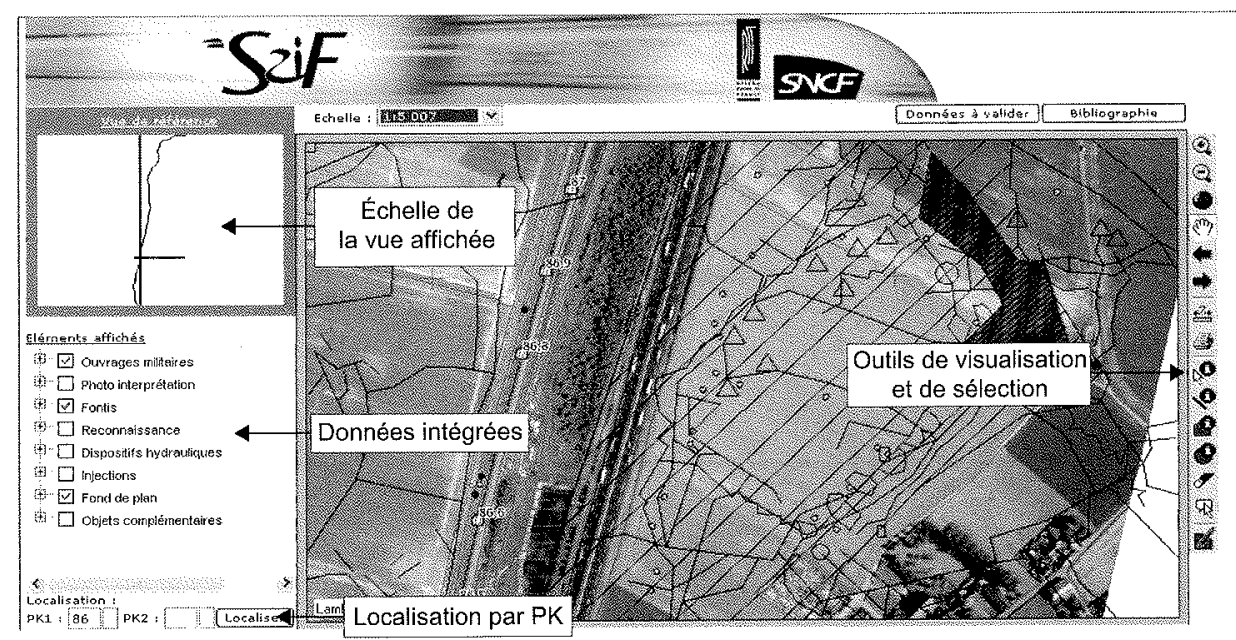

16. 3 Présentation des fonctionnalités à l'affichage du SIG.

GIS presentation of capabilities. 
- un cadre de localisation de l'information par rapport à l'ensemble du tracé ;

- une arborescence de couches d'informations affichables à l'écran que l'on active ou non suivant la nature de l'information désirée;

- un outil de sélection de points kilométriques ou de tronçon ;

- une barre de menu développant une quinzaine d'outils (zoom, sélection ponctuelle, sélection surfacique, édition de bases de données, impression) dont un outil de mise à jour uniquement accessible au gestionnaire technique du SIG, les utilisateurs n'ayant que lá possibilité de consulter les informations. Cette procédure permet d'assurer la cohérence des données saisies et leur uniformité. Ne sont par ailleurs accessibles aux utilisateurs que les données contrôlées et validées.

Les différentes couches d'informations ne sont affichables que sur une gamme d'échelles prédéfinies afin de ne pas saturer l'image de détails illisibles. Certaines informations, comme les investigations, précisent à grande échelle les linéaires investigués par chantier élémentaire et offrent, à une échelle plus fine, la carte de répartition des anomalies résiduelles et la position des sondages de contrôle. Hormis les fonds de plan, chaque couche d'informations est liée à une base de données (Figs. 4 et 5) qu'il est possible d'extraire de façon dynamique avec la zone requêtée (par exemple, le nombre de fontis recoupés par une droite ou inclus dans un périmètre tracé à l'écran sur le fond de plan...). De la même façon, il est possible d'accéder à des fichiers relatifs à l'information sélectionnée comme des rapports, des photographies, enregistrés informatiquement sous support standard.

Chaque nouveau fontis fait l'objet d'une fiche d'identification au format prédéfini (Fig. 6) et consultable en fichier joint. Ces fiches sont particulièrement utiles à l'exploitation et à l'amortissement des tournées de surveillance en permettant éventuellement d'affiner, si nécessaire, les conditions de déclenchement des tournées hélicoptères sur dépassement de seuil pluviométriques et l'efficacité de la couverture estimée des stations météorologiques de référence.

Enfin, des développements spécificues ont été réservés à l'éclition de support papier restituant automatiquement et à l'échelle désirée les fonds de plans reportant les couches d'informations sélectionnées (Fig. 7).

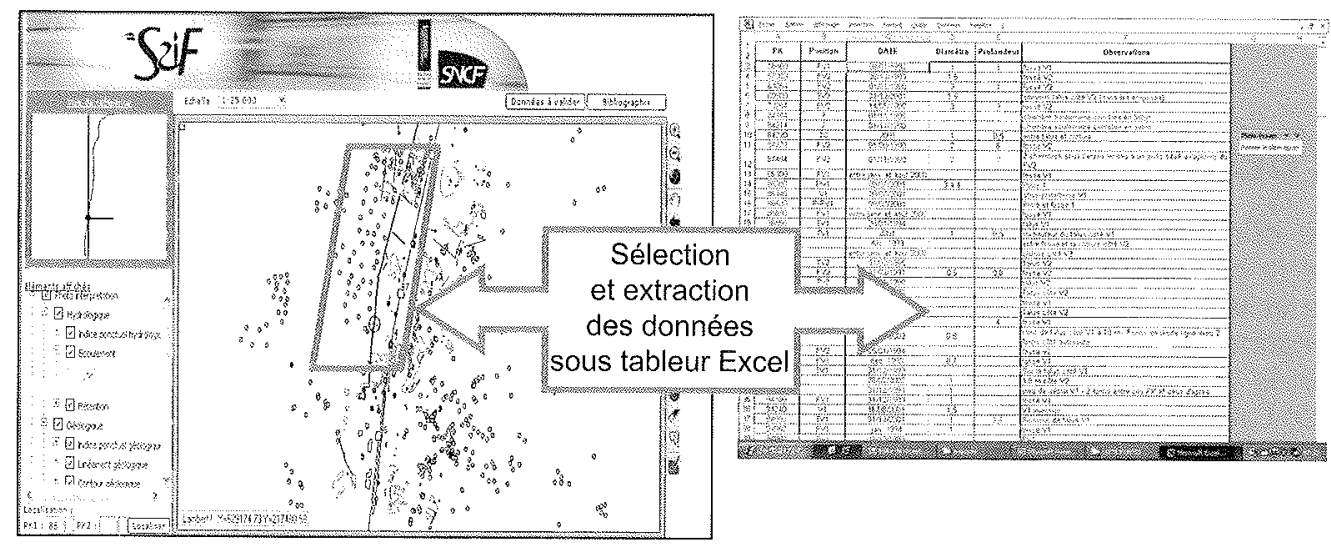

f. flc. 4 Consultation de bases de données sur critère de sélection.

Information extraction based on selection criteria.

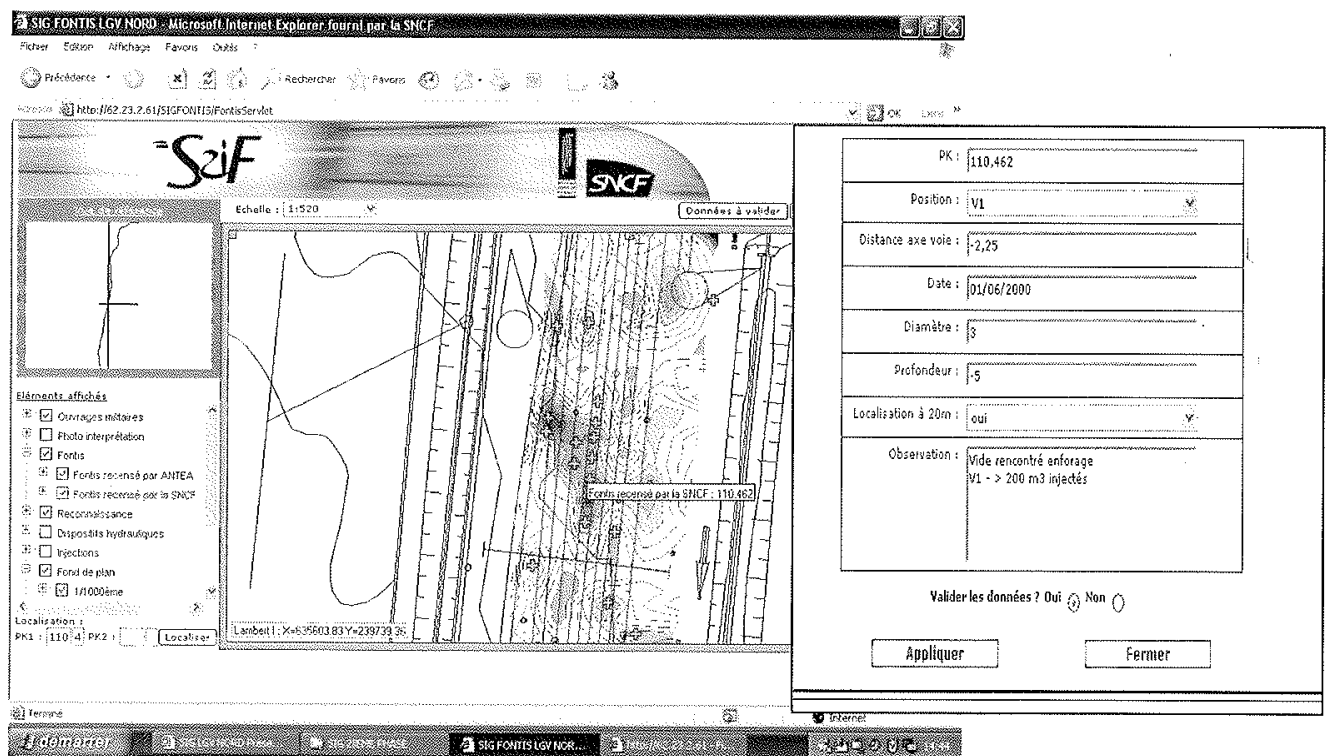

hos. Exemple de carte d'anomalie microgravimétrique et de localisation de sondages.

Example map of microgravimétric anomalies and borehole locations. 


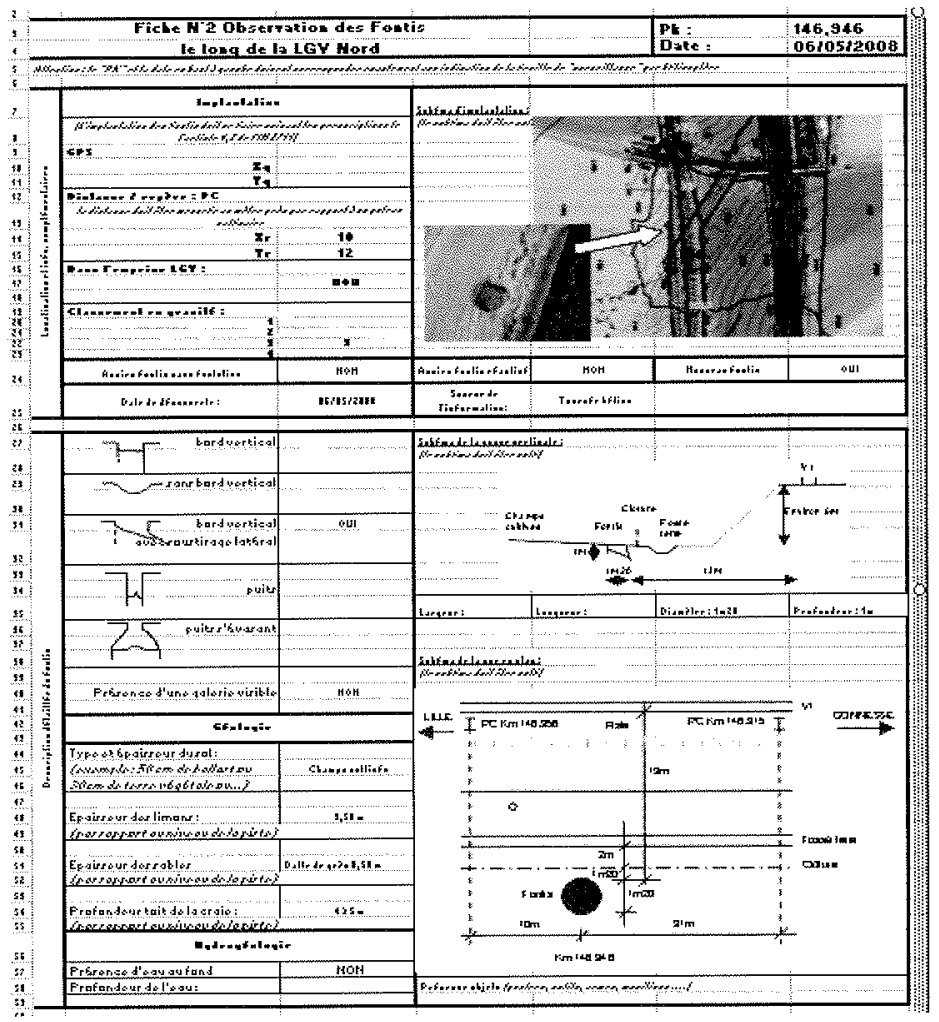

FG. 6 Exemple de fiche de signalement de fontis. Example of sinkhole identification form.

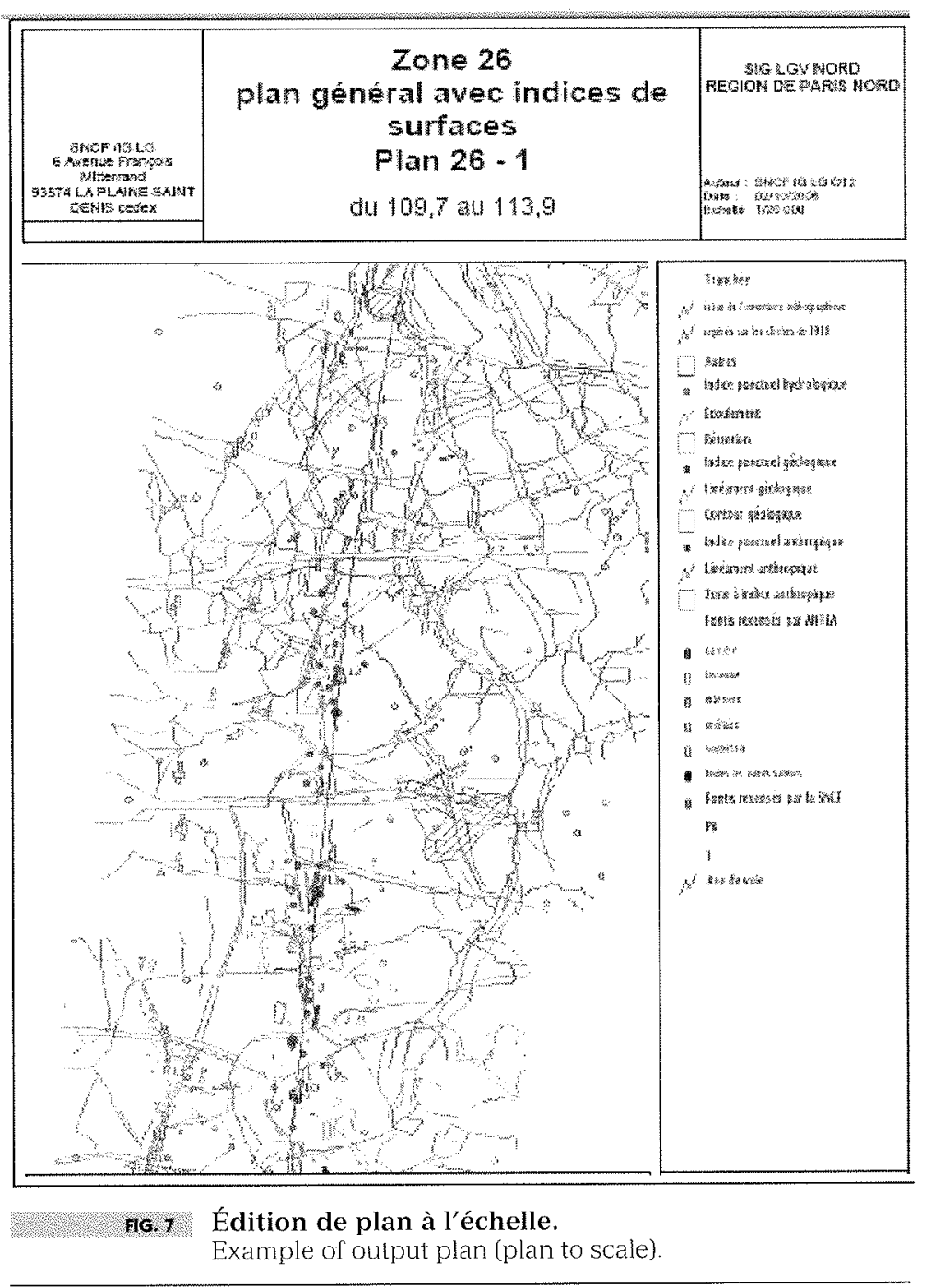




\section{Exemple de retour d'expérience du SIG depuis sa mise en application}

Depuis sa mise en application (janvier 2007), les conditions météorologiques ont été relativement clémentes en termes de précipitations. On dénombre moins d'une dizaine de fontis observés dans les emprises ou dans l'environnement proche du tracé recensés lors des tournées de surveillance. Lors de leur découverte, l'application SIG a montré sa forte contribution dans la prise de décision. Pour l'un d'entre eux, la consultation du SIG a permis instantanément de montrer qu'il s'agissait de tassement résiduel au niveau d'un ancien fontis en pied de talus de déblai ferroviaire coïncidant avec le tracé d'une ancienne tranchée militaire. La plateforme au droit de cet incident avait fait par ailleurs l'objet d'un chantier d'injection. Il n'y a donc pas eu de suite donnée en termes d'investigations ou de traitement, le secteur relevant d'une simple surveillance. Pour un autre cas, il s'agissait d'une petite dépression observée dans le ballast $(\varnothing 0,50 \mathrm{~m})$ et qui a conduit le service local à imposer une limitation de vitesse. Cette contrainte a pu être rapidement levée après mise en évidence de la présence d'un forage réalisé à cet emplacement précis pour auscultation vidéo. La zone ayant fait également l'objet d'un chantier d'injection, les désordres ont été imputés à un défaut de remplissage du forage à l'issue de l'auscultation vidéo. Cet exemple illustre la précision de l'information issue des archives et la forte réactivité offerte par le SIG.

\section{6}

\section{Conclusion}

En relation avec les enjeux, le récent développement des outils SIG ouvre de véritables perspectives dans le domaine de la maintenance de grandes infrastructures linéaires en accompagnement à la prise de décision. Concernant le domaine ferroviaire, cet outil s'avère particulièrement approprié pour l'ingénierie de maintenance, notamment dans le domaine de la gestion de patrimoine associée à une démarche d'analyse de risque.

\section{Bibliographie}

Méreau M. - Les systèmes d'informations (SIG) et la prise en compte de l'environnement dans les projets d'infrastructure. Revue générale des routes, $n^{\circ} 804$, mars 2002. 